\title{
Zukunftsplanung für Ärztinnen und Ärzte
}

\author{
Wie sehen meine finanziellen Möglichkeiten in 10 Jahren aus? Welche Mittel stehen mir nach meiner Pensionierung \\ monatlich zur Verfügung?
}

\section{P. Dahinden}

Korrespondenz: Patrik Dahinden, dipl. Treuhandexperte FMH Services Treuhand, Geschäftsstelle Sursee Lizenznehmerin:

Partner Treuhand AG, Sursee Christoph-Schnyder-Strasse 46 CH-6210 Sursee

Tel. 0419267045
Jeder von uns hat Pläne, Träume und Wünsche für die Zukunft. Sei es die Finanzierung eines Eigenheims, die Weltreise nach der Pensionierung, das Ferienhaus in der Toscana oder einfach die Ausbildung Ihrer Kinder. Alle diese Vorhaben benötigen Geld. Oft sind es daher auch genau diese Geldmittel, die unsere künftigen Möglichkeiten einschränken. Wer wissen will, wo seine Möglichkeiten und Grenzen in etwa liegen werden, sollte sich mit den folgenden Fragen genauer auseinandersetzen.

\section{Mit welchen Finanzmitteln darf ich planen?}

Die Analyse der bestehenden finanziellen Situation soll aufzeigen, welche Vermögenswerte bereits vorhanden sind. Nebst den «normalen» Vermögen wie z. B. Bankersparnissen, Wertschriften, Anlagefonds, Liegenschaften oder Lebensversicherungen müssen hier auch die künftigen Guthaben aus der staatlichen Altersvorsorge (AHV), der beruflichen Vorsorge (Pensionskasse) sowie der Säule 3a (gebundene Vorsorge) ermittelt werden.

$\mathrm{Zu}$ den bereits vorhandenen Mitteln müssen die künftigen Einkommen aus Erwerbstätigkeit, die Erträge aus den obigen Vermögenswerten und weiteren Einnahmequellen abgeschätzt werden. Bereits absehbare Veränderungen dieser Einnahmen sollten ebenfalls berücksichtigt werden. Diese können bei einer frühzeitigen Reduktion der Erwerbstätigkeit ebenso auftreten wie beim geplanten Wiedereinstieg des Ehepartners ins Erwerbsleben.

\section{Wie hoch ist mein künftiger Geldbedarf?}

Um den privaten Geldverbrauch für die Zukunft im voraus zu planen, ist ein Privatbudget das geeignete Hilfsmittel. Der wiederkehrende Mittelbedarf für Wohnen, Versicherungen, Fahrzeuge, Hobby, Steuern usw. lässt sich am besten anhand einer Liste mit den vorgegebenen Positionen realistisch ermitteln. Unter Umständen sind für verschiedene Lebensabschnitte auch unterschiedliche Budgets zu erstellen. So kann sich der Mittelbedarf beim Übertritt der Kinder in die finanzielle Unabhängigkeit entscheidend verändern oder die geplante Reduktion der Erwerbstätigkeit lässt den Geldbedarf für Hobbys merklich ansteigen.

Neben den wiederkehrenden Posten sind auch Geldabflüsse für einmalige Vorhaben wie z. B. den Umbau des Eigenheims, den geplanten Auslandsaufenthalt oder die Anschaffung Ihres Traumautos zu berücksichtigen.

\section{Zukunft optimieren}

Obwohl die oben ermittelten Werte für die Zukunft nur geschätzt werden können, gibt Ihnen eine jährliche Gegenüberstellung der Resultate wichtige Anhaltspunkte über Ihre finanzielle Zukunft bis über das Rentenalter hinaus.

Natürlich lassen sich in den meisten Fällen mit geeigneten Massnahmen Verbesserungen in Ihrer Zukunft erreichen. Sei es über einen planmässigen Aufbau Ihrer Vorsorge, das Ausnützen von steuerlichen Optimierungsmöglichkeiten, die Wahl zwischen Rentenleistungen oder Kapitalbezügen bei Ihrer Vorsorge und vieles mehr. Ihr Finanzberater wird Ihnen wertvolle Tipps und Anregungen geben können. Ihre Zukunft wird es Ihnen danken. 